$1988,37,4$

\title{
ON THE MASSLESS REPRESENTATIONS OF THE POINCARÉ GROUP
}

\author{
(Presented by H. Keres)
}

In this paper the systematic study of massless representations of the Poincaré group is given. When the finite dimensional representations of the homogeneous Lorentz group are used, the classification of massless representations differs from that given by Wigner. There exists only one necessary condition: the eigenvalues of the second Casimir operator must vanish. It appears that for a given representation all helicities are allowed.

\section{Introduction}

The important role of the Poincaré group in the field theory was discovered by $E$. P. Wigner $\left[{ }^{1}\right]$, who gives also the method for classification of irreducible representations known as the little group method. One of the possible realizations of physical representations, using Lorentz bispinors, was given in [2].

Proceeding from the Wigner analysis of Poincaré representations, S. Weinberg established the well-known result $\left[{ }^{3}\right]$ : when the irreducible representation $(k, l)$ of the homogeneous Lorentz group is used to describe massless states, one can have only $\lambda= \pm(k-l)$ helicities. The Weinberg theorem is analysed in the textbook [ $\left.{ }^{4}\right]$, where it is also stated that the possible helicities are limited to $\pm(k-l)$.

Recently the discussion of massless representation of the Poincaré group was given in $\left[{ }^{5,6}\right]$. In these papers the nature of Lorentz group representations of massless particles in the covariant field theory with indefinite metric is clarified and contrasted with that in the Wigner's theory. The authors come to the conclusion that in the covariant theory, finite-dimensional representations other than one-dimensional, are possible. They also prove that massless particles cannot be specified by the helicities only, but must be distinguished also by the types of fields describing them. It was stated $\left[{ }^{5,6}\right]$ that the requirement when the littlegroup translations must vanish, is not needed. It should be mentioned that this statement was also given in $\left[{ }^{7}\right]$.

In this paper we give a more systematic study of massless representations of the Poincare group in the covariant field theory where the finite dimensional representations of the homogeneous Lorentz group are used. When using the representations of the homogeneous Lorentz group, it is necessary that the eigenvalues of the second Casimir operator vanish. The condition that the little-group translations vanish, which leads to the Weinberg theorem, is not needed and may be treated as some subsidiary condition which separates certain specific helicities. In the general case for the representation $(k, l)$ all helicities $\lambda=k+l, \ldots,-(k+l)$ are allowed. The role of covariant wave equations in the field theory is to separate desired helicities. Therefore the general classification of massless representations in the covariant field theory is different from the Wigner's analysis, 


\section{Poincaré group}

The generators of the Poincaré group $M^{\mu v}, P^{v}$ satisfy the following commutation relations $\left[{ }^{1,2}\right]$

$$
\begin{gathered}
{\left[P^{\mu}, P^{v}\right]=0,} \\
{\left[M^{\mu v}, P^{\rho}\right]=\eta^{v \rho} P^{\mu}-\eta^{\mu \rho} P^{v},} \\
{\left[M^{\mu v}, M^{\rho \sigma}\right]=\eta^{v \rho} M^{\mu \sigma}+\eta^{\mu \sigma} M^{v \rho}-\eta^{\mu \rho} M^{v \sigma}-\eta^{v \sigma} M^{\mu \rho},}
\end{gathered}
$$

where $\eta^{\mu v}=\operatorname{diag}(+---)$. The eigenvalues of Casimir operators

$$
\begin{gathered}
P^{2}=P_{\mu} P^{\mu}, \\
W^{2}=W_{\mu} W^{\mu}=\frac{1}{2} P^{2} M^{\mu \nu} M_{\mu \nu}-P^{\mu} P_{v} M_{\mu \rho} M^{v \rho}
\end{gathered}
$$

label the irreducible representations of the Poincare group. Here $W^{\mu}$ is the Pauli-Lubanski pseudovector

$$
W^{\mu}=\frac{1}{2} \varepsilon^{\mu}{ }_{v \rho \sigma} P^{v} M^{\rho \sigma} .
$$

In physics more interesting are the massive representations when $P^{2}=$ $=m^{2}>0$ and the massless representations when $P^{2}=0$.

In this section we briefly recall the construction of massive representations. The construction of irreducible representations is more clear via the Wigner little-group method. Working in the momentum representation we have, when $P^{2}=m^{2}>0$, a system where the momentum takes the form

$$
\hat{p^{\mu}}=(\varepsilon m, 0,0,0),
$$

where $\varepsilon$ is the sign of energy $\varepsilon=p^{0} /\left|p^{0}\right|$. The little group of transformations $\hat{p}^{\mu}=R^{\mu} \hat{p}_{v}$, which leaves $\hat{p}^{\mu}$ unchanged, is the group of three dimensional rotations $\mathrm{SO}(3)$. It appears that in the rest of the system (2.5) the components of $W^{\mu}$ also satisfy the same commutation relations as the generators of the little group, and $W^{2}$ is related with the Casimir operator of the little group. Therefore the irreducible state at the rest $|(m, s) \hat{p} \sigma\rangle$ is defined in the following way

$$
\begin{aligned}
& P^{2}|(m, s) \hat{p} \sigma\rangle=m^{2}|(m, s) \hat{p} \sigma\rangle, \\
& W^{2}|(m, s) \hat{p} \sigma\rangle \equiv m^{2} S^{2}|(m, s) \hat{p} \sigma\rangle=m^{2} S(s+1)|(m, s) \hat{p} \sigma\rangle, \\
& S^{3}|(m, s) \hat{p} \sigma\rangle \equiv i M^{12}|(m, s) \hat{p} \sigma\rangle=\sigma|(m, s) \hat{p} \sigma\rangle .
\end{aligned}
$$

The irreducible representation is characterised by mass $m$ and spin $s$. The components of the corresponding state are labelled by the spin projection $\sigma$. The state of the system with arbitrary momentum $p^{\mu}=L^{\mu} \hat{p}^{v}$ is given by the boost transformation $U(L)$

$$
|(m, s) p \sigma\rangle=U(L)|(m, s) \hat{p} \sigma\rangle .
$$

\section{Massless representations}

In the massless case $P^{2}=0$ the same method of induced representations was used by Wigner in his pioneering work [ $\left.{ }^{1}\right]$. As we shall see in the following, the little group method does not work so well now as it did in the massive case. 
In the massless case we can take a system where

$$
\hat{p}^{\mu}=p(\varepsilon, 0,0,1) \text {. }
$$

Now the little group of transformations $\hat{p}^{\mu}=T^{\mu}{ }_{v} \hat{p}^{v}$ is the two-dimensional Euclidean group $\mathrm{E}(2)$. The components of $W^{\mu}$ in the $\hat{p}$ system are

$$
\begin{aligned}
& W^{0}=\varepsilon W^{3}=-p M^{12}, \\
& W^{1}=p\left(-\varepsilon M^{23}+M^{20}\right), \\
& W^{2}=p\left(-\varepsilon M^{31}+M^{01}\right) .
\end{aligned}
$$

It is easy to verify that the generators

$$
\begin{gathered}
w^{0}=M^{12}, \quad w^{1}=-\varepsilon M^{23}+M^{20}, \\
w^{2}=-\varepsilon M^{31}+M^{01}
\end{gathered}
$$

also satisfy the commutation relations of $\mathrm{E}(2)$

$$
\left[w^{1}, w^{2}\right]=0, \quad\left[w^{0}, w^{1}\right]=w^{2}, \quad\left[w^{0}, w^{2}\right]=-w^{1} .
$$

It follows from (3.4) that $w^{1}$ and $w^{2}$ are the generators of two-dimensional translations.

The second Casimir operator $W^{2}$ is expressed as

$$
W^{2}=-p^{2}\left(\left(w^{1}\right)^{2}+\left(w^{2}\right)^{2}\right) \text {. }
$$

The state $|\hat{p} \lambda\rangle$ of the $\hat{p}$ system satisfies $P^{2}|\hat{p} \lambda\rangle=0$. In order to obtain finite-dimensional representations, one must demand that the eigenvalue of $W^{2}$ also equals zero

$$
W^{2}|\hat{p} \lambda\rangle=0
$$

since $W^{2}$ depends on translation operators $w^{1}$ and $w^{2}$. In order to satisfy (3.6), one must require in addition that

$$
w^{1}|\hat{p} \lambda\rangle=w^{2}|\hat{p} \lambda\rangle=0
$$

In the massless case, as we have seen, the invariant operators $P^{2}$ and $W^{2}$ do not classify the different irreducible representations. Using the analogy with the massive case where $W^{\mu}$ give the generators of the little group, Wigner had demanded that the irreducible representations were classified by the irreducible representations of the little group. Since the translations of the little group vanish due to (3.7), the irreducible representations are classified by the eigenvalues of rotation operator $w^{0}$. Therefore the irreducible state is determined by

$$
S^{3}|\hat{p} \lambda\rangle \equiv i M^{12}|\hat{p} \lambda\rangle=\lambda|\hat{p} \lambda\rangle
$$

Thus, the irreducible representations are labelled by the helicity $\lambda$.

The classification of irreducible representations in the massless case is not so direct as it is in the massive case. Moreover, this classification does not follow directly from the invariants $P^{2}$ and $W^{2}$. For that reason it is not obligatory that the irreducible representations correspond to the single helicity $\lambda$. The photon, for example, has $\lambda= \pm 1$.

In this section we outlined the principles used for the general classification of massless representations. In physical applications the representations of the homogeneous Lorentz group - scalars, spinors, vectors, etc. are used, and on that basis one should construct the realization of Poincaré representations. As we shall demonstrate in the next section, the above-mentioned realization offers more flexibility. The requirement (3.7), for example, is not needed. 


\section{Realization of massless representations using Lorentz representations}

In the field theory the representations of the homogeneous Lorentz group are used. In order to construct the Poincaré representations, one must find the Casimir operators $P^{2}$ and $W^{2}$, and investigate their eigenvalues. As we shall see, this realization is somewhat specific and allows more flexibility.

Using the Lorentz field $\psi$ we have the following Poincaré generators in the momentum representation [ $\left.{ }^{2}\right]$

$$
P^{\mu}=p^{\mu}, \quad M^{\mu v}=L^{\mu v}+S^{\mu v},
$$

where $L^{\mu v}=p^{\mu} \partial / \partial p^{v}-p^{v} \partial / \partial p^{\mu}$ are the generators of the angular momentum and $S^{\mu v}$ are the generators of the Lorentz group corresponding to the field $\psi$.

$W^{\mu}$ and $W^{2}$ are now expressed by $p^{\mu}$ and $S^{\mu \nu}$ only

$$
\begin{gathered}
W^{\mu}=\frac{1}{2} \varepsilon^{\mu \rho \sigma} p^{v} S^{\rho \sigma}, \\
W^{2}=\frac{1}{2} p^{2} S^{\mu \nu} S_{\mu \nu}-p^{\mu} p_{v} S_{\mu \rho} S^{v \rho} .
\end{gathered}
$$

This fact turns out to be significant in the further considerations.

In the following part of this section we examine the same conditions that have been established in the analysis of massless representations by E.P. Wigner and obtain the result which is known as the Weinberg theorem $\left[{ }^{3}\right]$. i. e. if $\psi$ carries the irreducible representation $(k, l)$ of the homogeneous Lorentz group, only the helicities $\lambda= \pm(k-l)$ are allowed.

We shall give a simple proof of the Weinberg theorem using the canonical basis for Lorentz generators. This form of canonical basis was previously used in $\left[{ }^{7}\right]$.

Consider the irreducible field $\psi$ carrying the representation $(k, l)$. Following (3.7), we demand

$$
w^{1} \psi=w^{2} \psi=0
$$

Using (4.2), we obtain for $w^{0}, w^{1}, w^{2}$ in the $\hat{p}$ system

$$
w^{0}=S^{12}, \quad w^{1}=-\varepsilon S^{23}+S^{20}, \quad w^{2}=-\varepsilon S^{31}+S^{01} .
$$

In order to analyse the conditions (4.3), it is convenient to use the canonical basis for Lorentz generators, choosing the Cartan sub-algebra in the following way -

$$
H_{1}=S^{03}, \quad H_{2}=i S^{12} .
$$

The generators $E_{1}, \ldots, E_{4}$ are given by

$$
\begin{array}{ll}
E_{1}=v^{1}+i v^{2}, & E_{2}=v^{1}-i v^{2}, \\
E_{3}=v^{3}-i v^{4}, & E_{4}=v^{3}+i v^{4},
\end{array}
$$

where $v^{1}=\left.w^{1}\right|_{\varepsilon=1}, v^{2}=\left.w^{2}\right|_{\varepsilon=1}, v^{3}=\left.w^{1}\right|_{\varepsilon=-1}$ and $v^{4}=\left.w^{2}\right|_{\varepsilon=-1}$.

To any irreducible representation $(k, l)$ corresponds the weight diagram (Figure). The points inside the parallelogram $(h, \lambda)$ correspond to the components $\psi_{h \lambda}$, with $H_{1} \psi_{h \lambda}=h \psi_{h \lambda}$ and $H_{2} \psi_{h \lambda}=\lambda \psi_{h \lambda}$. All points are single. The action of generators $E_{1}, \ldots, E_{4}$ is the following -

$$
\begin{array}{ll}
E_{1} \psi_{h \lambda} \sim \psi_{h+1 \lambda+1}, & E_{2} \psi_{h \lambda} \sim \psi_{h+1 \lambda-1}, \\
E_{3} \psi_{h \lambda} \sim \psi_{h-1 \lambda-1,}, & E_{4} \psi_{h \lambda} \sim \psi_{h-1 \lambda+1},
\end{array}
$$


Now we turn to the analysis of conditions (4.3). Taking $\varepsilon=+1$ in (4.3) one has

$$
E_{1} \psi_{h \lambda}=E_{2} \psi_{h \lambda}=0 \text {. }
$$

Using (4.7) and Figure it is easy to verify that this condition is satisfied only for the right edge point

$$
h=h_{\max }=k+l, \quad \lambda=-(k-l) .
$$

Taking $\varepsilon=-1$ it follows from (4.3)

$$
E_{3} \psi_{h \lambda}=E_{4} \psi_{h \lambda}=0 \text {. }
$$

The last condition is satisfied only in the case of the left edge point

$$
h=h_{\min }=-(k+l), \quad \lambda=k-l .
$$

The results (4.9) and (4.11) prove the Weinberg theorem, i.e. $\lambda=$ $= \pm(k-l)$ if $(4.3)$ is satisfied. Using the Cartan basis for $S^{\mu v}$ we have also obtained that in the massless case the physical state is also the eigenstate of operator $H_{1}=\delta^{03}$.

Therefore, following the same procedure as in Section 3, we immediately arrive at the Weinberg's theorem. It should be mentioned that this theorem yields a severe restriction for the possible description of massless states. Photon, for example, cannot be described by the vector representation $(1 / 2,1 / 2)-A^{\mu}$, since then $\lambda=0 . \lambda= \pm 1$ helicities may be described by the antisymmetric tensor field $F^{\mu \nu}$ which carries $(1,0) \oplus(0,1)$.

In the next section we shall demonstrate that the condition (4.3) is not needed in general and may be treated as some additional restriction. This fact was already demonstrated in $\left[{ }^{7}\right]$, where it was also proved that the operators $w^{1}$ and $w^{2}$ have only zero eigenvalues.

\section{Massless representations which avoid the Weinberg theorem}

In the third section it was demonstrated that in the massless case the eigenvalue of the second Casimir operator $W^{2}$ must vanish. When using the Lorentz fields this condition must be preserved, therefore we demand

$$
W^{2} \psi=0 .
$$

In this section we demonstrate that the conditions (4.3) are not needed. At first we show that for $W^{2}$ there are only zero eigenvalues because $W^{2}$ is nilpotent and no additional conditions above (5.1) are needed.

The nilpotency of $W^{2}$ follows from the fact that it is expressed through the nilpotent operators $E_{1}, \ldots, E_{4}$. In the case of an irreducible representation $(k, l)$ we have $\left(E_{1,3}\right)^{2 l+1}=0$ and $\left(E_{2,4}\right)^{2 k+1}=0$. These conditions may be most easily verified using (4.7) and the weight diagram (Figure).

Weight diagram of the representation $(k, l)$, $k>l$.

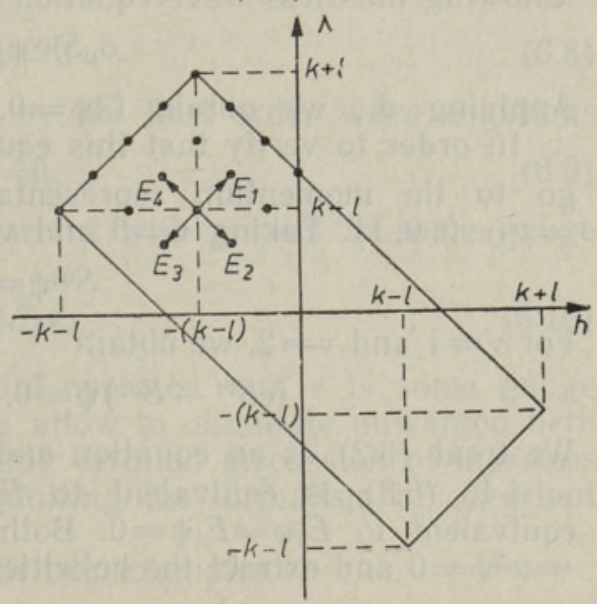


Using the $\hat{p}$ system we, in the $\varepsilon=+1$ case, may express $W^{2}$ as $W^{2}=$ $=-4 p^{2} E_{1} E_{2}$ and, in the $\varepsilon=-1$ case, as $W^{2}=-4 p^{2} E_{3} E_{4}$. Since $E_{1}, E_{2}$ and $E_{3}, E_{4}$ mutually commute, we have $\left(W^{2}\right)^{2 \alpha+1}=0$, where $\alpha=\min (k, l)$, which proves the nilpotency of $W^{2}$. Therefore $W^{2}$ has only zero eigenvalues.

In Section 3 the conditions $\omega^{1} \psi=w^{2} \psi=0$ were needed to give $W^{2} \psi=0$. In our case $W^{2} \psi=0$ follows from the nilpotency of $W^{2}$ and therefore no more additional conditions are needed. For that reason the Weinberg theorem must be treated as some additional condition which separates $\lambda= \pm(k-l)$ helicities.

Now we analyse what possibilities we have, demanding only (5.1): 1. If $\varepsilon=+1, W^{2} \psi=0$ gives, in the $\hat{p}$ system, $E_{1} E_{2} \psi=0$. In the weight diagram this conditions separates all the rightside edge points with helicities $\lambda=k+l, \ldots,-(k+l)$.

2. If $\varepsilon=-1, W^{2} \psi=0$ gives $E_{3} E_{4} \psi=0$. Now the same helicities are separated as in the previous case, but they correspond to all leftside edge points.

Therefore we have seen that in the case of representation $(k, l)$ all the helicities $\lambda=k+l, \ldots,-(k+l)$ are, in principle, allowed. In general they correspond to different eigenvalues of $H_{1}=S^{03}$ operator. In the case of representation $(1,1 / 2)$, for example, we have for $\varepsilon=+1$ the following possibilities: $h=-1 / 2, \quad \lambda=3 / 2 ; \quad h=1 / 2, \lambda=1 / 2$ and $-3 / 2 ; \quad h=3 / 2$, $\lambda=-1 / 2$. For a given eigenvalue $h$ there are one or two possible helicities. In order to extract the helicities corresponding to a given $h$, additional conditions are needed. In the field theory relativistic wave equations are playing the role of such conditions which can extract the desired helicities from a given representation.

In conclusion, we have arrived at the following result: using the Lorentz representations (scalars, spinors, ...) it is possible to describe all helicities. In order to extract the desired helicities, additional conditions must be used. In the next section we give two different types of wave equations. Following the Weinberg theorem it is possible to obtain a set of wave equations which describe $\lambda= \pm(k-l)$ helicities. The other set of massless wave equations is the set of gauge invariant equations, where the gauge freedom is used to describe needed helicities.

\section{Massless wave equations}

6.1. Wave equations and the Weinberg theorem. Using the irreducible representation $(k, l)$, it is possible to give the following massless wave equation $\left[{ }^{7}\right]$

$$
\partial_{\mu} S^{\mu v} \psi=(k+l) \partial^{v} \psi
$$

Applying $\partial_{v}$, we obtain $\square \psi=0$.

In order to verify that this equation describes helicities $\pm(k-l)$, we go to the momentum representation and use the system with $\hat{p}^{\mu}=$ $=p(\varepsilon, 0,0,1)$. Taking $v=0$ and $v=3$ in $(6.1)$, we obtain

$$
S^{03} \psi=\varepsilon(k+l) \psi \text {. }
$$

For $v=1$ and $v=2$, we obtain

$$
\left(\varepsilon S^{01}-S^{31}\right) \psi=0, \quad\left(\varepsilon S^{02}-S^{32}\right) \psi=0 .
$$

We treat (6.2) as an equation and (6.3) as subsidiary conditions. If $\varepsilon=$ $=+1,(6.3)$ is equivalent to $E_{1} \psi=E_{2} \psi=0$ and if $\varepsilon=-1$, (6.3) is equivalent to $E_{3} \psi=E_{4} \psi=0$. Both conditions are equivalent to $w^{1} \psi=$ $=w^{2} \psi=0$ and extract the helicities $\lambda= \pm(k-l)$. 
If, instead of the irreducible representation $(k, l)$ we use some reducible representation, the same considerations are valid. One can represent $\psi$ as a direct sum of irreducible representations $\left(k_{i}, l_{i}\right)$, and for each irreducible representation the helicities $\lambda_{i}= \pm\left(k_{i}-l_{i}\right)$ are separated.

The equation (6.1) was given in $\left[{ }^{8,9}\right]$, and recently in [ $\left.{ }^{10}\right]$. In the special case of representations $(s, 0)$ and $(0, s)$ similar equations were given in $\left.{ }^{3}\right]$ and ${ }^{[11}$. It should be mentioned that $V$. Bargmann and E. P. Wigner proposed in $\left[{ }^{2}\right]$ the following equation

$$
W^{\mu} \psi=i(k-l) \partial^{\mu} \psi,
$$

which in $\left[{ }^{10}\right]$ was given in the following form

$$
\partial_{\mu} \bar{S}^{\mu v} \psi=(k-l) \partial^{v} \psi,
$$

where $\bar{S}^{\mu v}=\frac{1}{2} \varepsilon^{\mu v}{ }_{\rho \sigma} S^{\rho \sigma}$. It is possible to verify that $(6.1)$ and $(6.5)$ are equivalent, if $k \neq l$.

We conclude this Section with two examples.

1. Representation $(1 / 2,1 / 2)$. Using the vector field $A^{\mu}$ and the corresponding generators $S^{\mu v}$, we obtain from (6.1)

$$
\partial^{\mu} A^{v}-\partial^{v} A^{\mu}-\eta^{\mu v} \partial_{\rho} A^{\rho}=0 .
$$

Equation (6.6) describes $\lambda=0$.

2. Representation $(\mathbf{1}, 0) \oplus(0,1)$. Using the antisymmetrical tensor field $F^{\mu v}$ we obtain the equation

$$
\partial^{\rho} F^{\mu v}+\partial^{v} F^{\rho \mu}+\partial^{\mu} F^{v \rho}+\eta^{\rho \mu} \partial_{\sigma} F^{\sigma v}-\eta^{\rho v} \partial_{\sigma} F^{\sigma \mu}=0 .
$$

This set of equations is equivalent to the Maxwell equations and therefore describes $\lambda= \pm 1$.

It should be remarked that the construction of Lagrangians corresponding to (6.1) is more complicated. Due to the additional vector index in (6.1), it is not easy to find a correct Lagrangian and to develop the corresponding Lagrangian field theory. In the case of antisymmetrical tensor field $F^{\mu v}$ an attempt to use the vector Lagrangian was made in [12]. An additional vector $\lambda^{\mu}$, satisfying $\lambda^{2} \neq 0$, was introduced in $\left[{ }^{13}\right]$, and the Lagrangian $L=\lambda_{v}\left(\partial_{\mu} S^{\mu v}-(k+l) \partial^{v}\right) F$ for $F^{\mu v}$ was proposed. Similar Lagrangian can be used in the case of all equations (6.1). However, the physical meaning of $\lambda^{\mu}$ vector is not completely clear.

6.2. Gauge invariant wave equations. The second and mostly used possibility to describe massless field is to exploit the gauge invariant wave equations. The boson fields are usually described by the second order wave equations

$$
\partial_{\mu} \partial_{v} \beta^{\mu v} \psi=0
$$

and the fermion fields are described by the first order wave equations

$$
\partial_{\mu} \beta^{\mu} \psi=0 \text {. }
$$

In both cases the equation is invariant with respect to some gauge transformation

$$
\delta \psi=f(\partial) \varepsilon,
$$

where $f(\partial)$ is a first order differential operator and $\varepsilon$ is some gauge parameter. The gauge transformations allow to eliminate unwanted helicities. In this paper we do not give any detailed discussion of massless gauge fields. Some recent results exploiting the formalism of covariant spin projectors are given in $\left[{ }^{14,15}\right]$.

This Section we also conclude with two examples, 
1. Representation $(1 / 2,1 / 2)$. The gauge invariant wave equation for $A^{\mu}$ is the following -

$$
\square A^{\mu}-\partial^{\mu} \partial_{v} A^{v}=0 .
$$

Equation (6.11) is invariant with respect to gauge transformation

$$
\delta A^{\mu}=\partial^{\mu} \varepsilon,
$$

where $\varepsilon$ is a scalar field. Equation (6.11) is the well-known field equation, describing the electromagnetic field with helicities \pm 1 .

2. Representation $(\mathbf{1}, \mathbf{0}) \oplus(\mathbf{0 , 1})$. The antisymmetrical tensor field $F^{\mu v}$ allows to obtain two gauge invariant equations both describing $\lambda=0$. The first equation

$$
\square F^{\mu v}-\partial^{\mu} \partial_{\rho} F^{\rho v}+\partial^{v} \partial_{\rho} F^{\rho \mu}=0
$$

is invariant with respect to the gauge transformation

$$
\delta F^{\mu v}=\partial^{\mu} \varepsilon^{v}-\partial^{v} \varepsilon^{\mu},
$$

where $\varepsilon^{\mu}$ is a vector field. The second equation

$$
\partial^{\mu} \partial_{\rho} F^{\rho v}-\partial^{v} \partial_{\rho} F^{\rho \mu}=0
$$

is invariant with respect to

$$
\delta F^{\mu v}=\varepsilon^{\mu v \rho}{ }_{\sigma} \partial_{\rho} \varepsilon^{\sigma} .
$$

The first equation (6.13) was given in $\left[{ }^{16}\right]$, where an object described by $F^{\mu v}$, was called «notoph» in contrast to photon described by the Maxwell equations (6.7). Both (6.13) and (6.15) follow from a single general gauge invariant equation for $F^{\mu v}$.

These examples demonstrate that depending on the field equation one can describe different helicities. In the case of vector field $A^{\mu}$ it is possible to describe $\lambda=0$ or $\lambda= \pm 1$ the same helicities can be described by using antisymmetrical tensor field $F^{\mu v}$. The given examples are in accordance with the general condition $W^{2} \psi=0$.

\section{Conclusions}

In this paper the general description of massless states in the field theory is analysed. It appears that the general classification of massless representations of the Poincaré group differs from the standard one due to using the representations of the homogeneous Lorentz group. The second Casimir operator $W^{2}$ is expressed by Lorentz generators $S^{\mu v}$ which are finite-dimensional matrices. For this reason $W^{2}$ is nilpotent and therefore one must demand only $W^{2} \psi=0$. The last condition allows different helicities to be described using the field $\psi$. In order to describe states with fixed helicities invariant wave equations are needed. One set that has been discussed satisfies the Weinberg theorem. The other set, which consists of gauge invariant equations, describes helicities that are not allowed by the Weinberg theorem. The main result is that in the case of representation $(k, l)$ all helicities $\lambda=k+l, \ldots,-(k+l)$ are possible.

In conclusions it should be remarked that there are some more possible realizations of massless states. In the $\lambda=0$ case the following first order realization is possible

$$
\partial^{\mu} \varphi=A^{\mu}, \quad \partial_{\mu} A^{\mu}=0,
$$


This realization can be expressed in the general form as

$$
\partial_{\mu} \beta^{\mu} \psi=\beta \psi,
$$

where $\beta$ is a singular matrix. Equations in this form were first discussed in $\left[{ }^{17}\right]$ and afterwards they have also been proposed in $\left[{ }^{18}\right]$. Recently the Fronsdal massless equations were given in this form in $\left.{ }^{19}\right]$.

\section{RFFERENCES}

1. Wigner, E. P. Ann. Math., 40, 149-204 (1939).

2. Bargmann, V., Wigner, E. P. Proc. Nat. Acad. Sci., 34, № 5, $211-223$ (1948).

3. Weinberg, S. Phys. Rev., 134, № 5, B882-896 (1964).

4. Румер Ю. Ю., Фет А. М. Теория групп и квантованные поля. М., «Наука», 1977 (\$ 19).

5. Kugo, T., Uehara, S. Progr. Theor. Phys., 66, № 3, $1044-1060$ (1981).

6. Kugo, T. Phys. Lett., 109B, № 3, 205-208 (1982).

7. Loide, R.-K. Preprint FAI-19. Tartu, 1972.

8. Niederer, U. H., O’Raifeartaigh, L. Fortschr. Phys., 22, № 3, 131-157 (1974).

9. Niederer, $U$. Fortschr. Phys., 27, № 4, 191-207 (1979).

10. Степановский Ю. П. Теор. и мат. физ., 47, № 3, 343-351 (1981).

11. Степановский Ю. П. Укр. физ. ж., 9, № 11, 1165-1168 (1964).

12. Sudbery, A. J. Phys. A: Math. Gen., 19, № 2, L33-L36 (1986).

13. Кривский И. Ю., Симулик В. М. Препринт ИЯИ АН УССР. КИЯИ-86-41, 1986.

14. Loide, R.-K., Polt, A. Proc. Acad. Sci. ESSR. Phys. Math., 35, № 1, 43-55 (1986).

15. Loide, R.-K. J. Phys. A: Math. Gen., 19, № 5, $811-820$ (1986).

16. Огиевецкий В. Н., Полубаринов Н. В. Ядер. физика, 4, вып. 1, $216-224$ (1966),

17. Harish-Chandra Proc. Roy Soc., A186, 502 (1946).

18. Федоров Ф. Н. ДАН СССР, 82, № 1, 37-40 (1952).

19. Cox, W. J. Phys. A: Math. Gen., 16, № 10, 2331-2345 (1983).

Academy of Sciences of the Estonian SSR, Institute of Physics

Received

Tallinn Technical University

June 12,1987

M. KOIV, R.-K. LOIDE, I. OTS, R. SAAR

\section{POINCARÉ RUHMA MASSITA ESITUSTEST}

On antud Poincaré rühma massita esituste süstemaatiline analüüs. Homogeense Lorentzi rühma lõplikdimensiooniliste esituste kasutamisel erineb massita esituste klassifitseerimine Wigneri analüüsist. Sel juhul on ainult üks tarvilik tingimus: teise Casimiri operaatori omaväärtuste vōrdumine nulliga. Võib kirjeldada spiraalsusi, mis rahuldavad Weinbergi teoreemi, kuid ka kõiki teisi spiraalsusi.

М. КЫИВ, Р.-К. ЛОЙДЕ, И. ОТС, Р. СААР

\section{О БЕЗМАССОВЫХ ПРЕДСТАВЛЕНИЯХ ГРУППЫ ПУАНКАРЕ}

Проведено систематическое исследование безмассовых представлений группы Пуанкаре. Когда применяются конечномерные представления однородной группы Лоренца, классификация безмассовых представлений отличается от анализа Вигнера. Имеется только одно необходимое условие - равенство нулю собственных значений второго оператора Казимира. Можно описывать спиральности, удовлетворяющие теореме Вайнберга, но н все остальные спиральности. 